\title{
On the multiplicity of solutions for a kind of fourth-order equation depending on two real parameters
}

\section{Armin Hadjian ${ }^{1 *}$ (D) and Maryam Ramezani ${ }^{1}$}

*Correspondence:

hadjian83@gmail.com

${ }^{1}$ Department of Mathematics,

Faculty of Basic Sciences, University

of Bojnord, P.O. Box 1339, Bojnord

94531, Iran

\begin{abstract}
In this paper, by suitable assumptions on nonlinear boundary term, we establish the existence of three distinct weak solutions for a kind of fourth-order boundary value problem depending on two parameters.
\end{abstract}

MSC: 34B15; 58E05

Keywords: Fourth-order equations; Critical point theory; Variational methods; Three solutions

\section{Introduction}

In this paper, we consider the following fourth-order problem:

$$
\left\{\begin{array}{l}
u^{(i v)}(x)=\lambda f(x, u(x)), \quad \text { in }[0,1], \\
u(0)=u^{\prime}(0)=0, \\
u^{\prime \prime}(1)=0, \quad u^{\prime \prime \prime}(1)=\mu g(u(1)),
\end{array}\right.
$$

where $\lambda, \mu \in] 0, \infty\left[, g: \mathbb{R} \rightarrow \mathbb{R}\right.$ is a continuous function and $f:[0,1] \times \mathbb{R} \rightarrow \mathbb{R}$ is an $L^{1}$ Caratéodory function.

Problem (1.1) describes the static equilibrium of a flexible elastic beam of length 1 when, along its length, a load $f$ is added to cause deformation. Precisely, conditions $u(0)=u^{\prime}(0)=$ 0 mean that the left end of the beam is fixed and conditions $u^{\prime \prime}(1)=0, u^{\prime \prime \prime}(1)=\mu g(u(1))$ mean that the right end of the beam is attached to a bearing device, given by the function $g$.

Existence and multiplicity of solutions for fourth-order boundary value problems have been discussed by several authors in the last decades; see for example $[1,4,5,9-13,16$, $17,19-21]$ and the references therein.

In particular, Yang et al. [21] used Ricceri's variational principle [18] to establish the existence of at least two classical solutions generated from $g$ for problem (1.1) with $\mu=1$.

(c) The Author(s) 2021. This article is licensed under a Creative Commons Attribution 4.0 International License, which permits use, sharing, adaptation, distribution and reproduction in any medium or format, as long as you give appropriate credit to the original author(s) and the source, provide a link to the Creative Commons licence, and indicate if changes were made. The images or other third party material in this article are included in the article's Creative Commons licence, unless indicated otherwise in a credit line to the material. If material is not included in the article's Creative Commons licence and your intended use is not permitted by statutory regulation or exceeds the permitted use, you will need to obtain permission directly from the copyright holder. To view a copy of this licence, visit http://creativecommons.org/licenses/by/4.0/. 
The authors in [9], using a multiplicity result by Cabada and Iannizzotto [8], ensured the existence of at least two nontrivial classical solutions for the problem

$$
\left\{\begin{array}{l}
u^{(4)}(x)+\lambda f(x, u(x))=0, \quad 0<x<1, \\
u(0)=u^{\prime}(0)=u^{\prime \prime}(1)=0, \\
u^{\prime \prime \prime}(1)=\lambda g(u(1))
\end{array}\right.
$$

where the functions $f:[0,1] \times \mathbb{R} \rightarrow \mathbb{R}$ and $g: \mathbb{R} \rightarrow \mathbb{R}$ are continuous and $\lambda \geq 0$ is a real parameter.

Bonanno et al. [4], by means of an abstract critical points result of Bonanno [2], studied the existence of at least one nonzero classical solution for problem (1.1).

In [12], by using a smooth version of [7, Theorem 2.1], Heidarkhani et al. established the existence of infinitely many generalized solutions for the following perturbed fourth-order problem:

$$
\left\{\begin{array}{l}
u^{(4)}(t)=\lambda f(t, u(t))+\mu g(t, u(t))+p(u(t)), \quad 0<t<1, \\
u(0)=u^{\prime}(0)=0, \\
u^{\prime \prime}(1)=0, \quad u^{\prime \prime \prime}(1)=h(u(1)),
\end{array}\right.
$$

where $\lambda>0, \mu \geq 0$ are two parameters, $f, g$ are two $L^{2}$-Caratéodory functions, and $p, h$ are Lipschitz continuous functions such that $p(0)=h(0)=0$.

Also in [11], the present authors obtained sufficient conditions to guarantee that problem (1.1) has infinitely many classical solutions.

More recently, Heidarkhani and Gharehgazlouei [13], using an immediate consequence of [3, Theorem 3.3], ensured the existence of at least three generalized solutions for the problem

$$
\left\{\begin{array}{l}
u^{(i v)}(x)=\lambda f(x, u(x))+h(u(x)), \quad \text { in }[0,1] \\
u(0)=u^{\prime}(0)=u^{\prime \prime}(1)=0 \\
u^{\prime \prime \prime}(1)=\mu g(u(1))
\end{array}\right.
$$

where $\lambda>0, \mu \geq 0$ are two parameters, $f:[0,1] \times \mathbb{R} \rightarrow \mathbb{R}$ is an $L^{1}$-Caratéodory function, $g: \mathbb{R} \rightarrow \mathbb{R}$ is a nonnegative continuous function, and $h: \mathbb{R} \rightarrow \mathbb{R}$ is a Lipschitz continuous function such that $h(0)=0$.

Motivated by the above works, the aim of the present paper is to offer the existence of three solutions for fourth-order problem (1.1) by using two kinds of critical point theorems obtained in $[3,6]$.

For completeness, we cite the recent and nice works $[14,15]$ as general references on the subject treated in this paper.

\section{Abstract setting}

In order to study problem (1.1), the variational setting is the space

$$
X:=\left\{u \in H^{2}([0,1]): u(0)=u^{\prime}(0)=0\right\},
$$


where $H^{2}([0,1])$ is the Sobolev space of all function $u:[0,1] \rightarrow \mathbb{R}$ such that $u$ and its distributional derivative $u^{\prime}$ are absolutely continuous and $u^{\prime \prime}$ belongs to $L^{2}([0,1]) . X$ is a Hilbert space with the inner product

$$
\langle u, v\rangle:=\int_{0}^{1} u^{\prime \prime}(t) v^{\prime \prime}(t) d t
$$

and the corresponding norm

$$
\|u\|:=\left(\int_{0}^{1}\left(u^{\prime \prime}(t)\right)^{2} d t\right)^{\frac{1}{2}}
$$

We observe that the norm $\|\cdot\|$ on $X$ is equivalent to the usual norm

$$
\int_{0}^{1}\left(|u(t)|^{2}+\left|u^{\prime}(t)\right|^{2}+\left|u^{\prime \prime}(t)\right|^{2}\right) d t
$$

It is well known that the embedding $X \hookrightarrow C^{1}([0,1])$ is compact and

$$
\|u\|_{C^{1}([0,1])}:=\max \left\{\|u\|_{\infty},\left\|u^{\prime}\right\|_{\infty}\right\} \leq\|u\|
$$

for all $u \in X$ (see [21]).

We say that $u \in X$ is a weak solution of problem (1.1) whenever

$$
\int_{0}^{1} u^{\prime \prime}(x) v^{\prime \prime}(x) d x-\lambda \int_{0}^{1} f(x, u(x)) v(x) d x+\mu g(u(1)) v(1)=0
$$

for all $v \in X$. By a classical solution of problem (1.1) we mean a function $u \in C^{1}([0,1])$ such that $u^{(i v)}(x) \in C([0,1])$ and the boundary conditions and the equation are satisfied in $[0,1]$.

Our main tools are critical point theorems that we recall here in a convenient form. The first result has been obtained in [6], and it is a more precise version of Theorem 3.2 of [3]. The second one has been established in [3].

Lemma 2.1 ([6, Theorem 3.6]) Let $X$ be a reflexive real Banach space; $\Phi: X \rightarrow \mathbb{R}$ be a coercive, continuously Gâteaux differentiable, and sequentially weakly lower semicontinuous functional whose Gâteaux derivative admits a continuous inverse on $X^{*} ; \Psi: X \rightarrow \mathbb{R}$ be a continuously Gâteaux differentiable functional whose Gâteaux derivative is compact such that

$$
\Phi(0)=\Psi(0)=0
$$

Assume that there exist $r>0$ and $\bar{x} \in X$, with $r<\Phi(\bar{x})$, such that

$\left(\mathrm{a}_{1}\right) \frac{\sup _{\Phi(x) \leq r} \Psi(x)}{r}<\frac{\Psi(\bar{x})}{\Phi(\bar{x})}$;

$\left(\mathrm{a}_{2}\right)$ for each $\left.\lambda \in \Lambda_{r}:=\right] \frac{\Phi(\bar{x})}{\Psi(\bar{x})}, \frac{r}{\sup _{\Phi(x) \leq r} \Psi(x)}[$, the functional $\Phi-\lambda \Psi$ is coercive.

Then, for each $\lambda \in \Lambda_{r}$, the functional $\Phi-\lambda \Psi$ has at least three distinct critical points in $X$.

Lemma 2.2 ([3, Theorem 3.3]) Let $X$ be a reflexive real Banach space; $\Phi: X \rightarrow \mathbb{R}$ be a convex, coercive, and continuously Gâteaux differentiable functional whose derivative admits 
a continuous inverse on $X^{*} ; \Psi: X \rightarrow \mathbb{R}$ be a continuously Gâteaux differentiable functional whose derivative is compact such that

$$
\inf _{X} \Phi=\Phi(0)=\Psi(0)=0
$$

Assume that there are two positive constants $r_{1}, r_{2}$ and $\bar{x} \in X$, with $2 r_{1}<\Phi(\bar{x})<\frac{r_{2}}{2}$, such that

(b $\left.b_{1}\right) \frac{\sup _{\Phi(x)<r_{1}} \Psi(x)}{r_{1}}<\frac{2}{3} \frac{\Psi(\bar{x})}{\Phi(\bar{x})}$;

(b) $\frac{\operatorname{bup}_{\Phi}(x)<r_{2} \Psi(x)}{r_{2}}<\frac{1}{3} \frac{\Psi(\bar{x})}{\Phi(\bar{x})}$

$\left(\mathrm{b}_{3}\right)$ for each $\lambda$ in

$$
\left.\Lambda^{\prime}:=\right] \frac{3}{2} \frac{\Phi(\bar{x})}{\Psi(\bar{x})}, \min \left\{\frac{r_{1}}{\sup _{\Phi(x)<r_{1}} \Psi(x)}, \frac{\frac{r_{2}}{2}}{\sup _{\Phi(x)<r_{2}} \Psi(x)}\right\}[
$$

and for every $x_{1}, x_{2} \in X$, which are local minima for the functional $\Phi-\lambda \Psi$ and such that $\Psi\left(x_{1}\right) \geq 0$ and $\Psi\left(x_{2}\right) \geq 0$, one has $\inf _{s \in[0,1]} \Psi\left(s x_{1}+(1-s) x_{2}\right) \geq 0$.

Then, for each $\lambda \in \Lambda^{\prime}$, the functional $\Phi-\lambda \Psi$ has at least three distinct critical points which lie in $\Phi^{-1}(]-\infty, r_{2}[)$.

We use the following notations:

Corresponding to $f$, $g$, we introduce the functions $F, G$ as follows:

$$
F(x, \xi):=\int_{0}^{\xi} f(x, t) d t, \quad G(\xi):=-\int_{0}^{\xi} g(t) d t
$$

for all $x \in[0,1]$ and $\xi \in \mathbb{R}$. Also, for each $\theta$ and $\eta$ of positive real numbers, define

$$
F^{\theta}=\int_{0}^{1} \sup _{|\xi| \leq \theta} F(x, \xi) d x, \quad G^{\theta}=\sup _{|\xi| \leq \theta} G(\xi), \quad G_{\eta}=\inf _{|\xi| \leq \eta} G(\xi) .
$$

\section{Main results}

In this section, we present our main result on the existence of at least three weak solutions for problem (1.1).

In order to introduce our result, we fix $\theta, \eta>0$ such that

$$
\frac{32 \pi^{4} \eta^{2}}{27 \int_{\frac{3}{4}}^{1} F(x, \eta) d x}<\frac{\theta^{2}}{2 F^{\theta}},
$$

and pick

$$
\Lambda:=] \frac{32 \pi^{4} \eta^{2}}{27 \int_{\frac{3}{4}}^{1} F(x, \eta) d x}, \frac{\theta^{2}}{2 F^{\theta}}[.
$$

Set

$$
\delta:=\min \left\{\frac{\theta^{2}-2 \lambda F^{\theta}}{2 G^{\theta}}, \frac{\frac{32}{27} \pi^{4} \eta^{2}-\lambda \int_{\frac{3}{4}}^{1} F(x, \eta) d x}{G(\eta)}\right\}
$$


and

$$
\bar{\delta}:=\min \left\{\delta, \frac{1}{\max \left\{0,4 \lim \sup _{|\xi| \rightarrow+\infty} \frac{G(\xi)}{\xi^{2}}\right\}}\right\},
$$

where we read $r / 0=+\infty$. For instance, $\bar{\delta}=+\infty$ when $\lim \sup _{|\xi| \rightarrow+\infty} \frac{G(\xi)}{\xi^{2}} \leq 0$ and $G(\eta)=$ $G^{\theta}=0$.

With the above notations we are able to prove the following multiplicity property.

Theorem 3.1 Assume that there exist two positive constants $\theta$, $\eta$, with $\theta<\frac{8}{3 \sqrt{3}} \pi^{2} \eta$, such that

(A1) $F(x, t) \geq 0$ for each $(x, t) \in\left[\frac{3}{8}, \frac{3}{4}\right] \times[0, \eta]$;

(A2) $\frac{F^{\theta}}{\theta^{2}}<\frac{27}{64 \pi^{4}} \frac{\int_{\frac{3}{4}}^{1} F(x, \eta) d x}{\eta^{2}}$;

(A3) $\lim \sup _{|\xi| \rightarrow+\infty} \frac{\sup _{x \in[0,1]} F(x, \xi)}{\xi^{2}}<\frac{F^{\theta}}{2 \theta^{2}}$.

Then, for every $\lambda \in \Lambda$, where $\Lambda$ is given by (3.1), and for every continuous function $g: \mathbb{R} \rightarrow \mathbb{R}$ such that

$$
\limsup _{|\xi| \rightarrow+\infty} \frac{G(\xi)}{\xi^{2}}<+\infty
$$

there exists $\bar{\delta}>0$ given by (3.3) such that, for each $\mu \in[0, \bar{\delta}[$, problem (1.1) admits at least three distinct weak solutions.

Proof Our aim is to apply Lemma 2.1 to problem (1.1). To this end, we introduce the functionals $\Phi, \Psi: X \rightarrow \mathbb{R}$ as follows:

$$
\begin{aligned}
& \Phi(u):=\frac{1}{2}\|u\|^{2}, \\
& \Psi(u):=\int_{0}^{1} F(x, u(x)) d x+\frac{\mu}{\lambda} G(u(1)) .
\end{aligned}
$$

It is well known that $\Psi$ is a differentiable functional whose differential at the point $u \in X$ is

$$
\Psi^{\prime}(u)(v)=\int_{0}^{1} f(x, u(x)) v(x) d x-\frac{\mu}{\lambda} g(u(1)) v(1)
$$

for every $v \in X$. Moreover, in [21], the authors proved that $\Psi^{\prime}$ is strongly continuous on $X$, which implies that $\Psi^{\prime}$ is a compact operator. Furthermore, by standard arguments, $\Phi$ is coercive and continuously differentiable whose differential at the point $u \in X$ is

$$
\Phi^{\prime}(u)(v)=\int_{0}^{1} u^{\prime \prime}(x) v^{\prime \prime}(x) d x
$$

for each $v \in X$. Also, in [21] it is proved that $\Phi^{\prime}$ admits a continuous inverse on $X^{*}$. Moreover, $\Phi$ is sequentially weakly lower semicontinuous. One can show that the weak solutions of problem (1.1) are exactly the solutions of the equation $\Phi^{\prime}(u)-\lambda \Psi^{\prime}(u)=0$. 
Fix $\lambda \in \Lambda$ and put $r=\frac{\theta^{2}}{2}$. Then, for $u \in X$ with $\Phi(u) \leq r$,

$$
\begin{aligned}
\sup _{\Phi(u) \leq r} \Psi(u) & =\sup _{\Phi(u) \leq r}\left(\int_{0}^{1} F(x, u(x)) d x+\frac{\mu}{\lambda} G(u(1))\right) \\
& \leq \int_{0}^{1} \sup _{|t| \leq \theta} F(x, t) d x+\frac{\mu}{\lambda} G^{\theta}=F^{\theta}+\frac{\mu}{\lambda} G^{\theta} .
\end{aligned}
$$

Therefore,

$$
\frac{\sup _{\Phi(u) \leq r} \Psi(u)}{r} \leq \frac{2 F^{\theta}}{\theta^{2}}+\frac{2 \mu}{\lambda} \frac{G^{\theta}}{\theta^{2}}
$$

From this, if $G^{\theta}=0$, it is clear that

$$
\frac{\sup _{\Phi(u) \leq r} \Psi(u)}{r}<\frac{1}{\lambda}
$$

while if $G^{\theta}>0$, it turns out to be true bearing in mind that

$$
\mu<\frac{\theta^{2}-2 \lambda F^{\theta}}{2 G^{\theta}} .
$$

Denote by $w$ the function of $X$ defined by

$$
w(x):= \begin{cases}0, & x \in\left[0, \frac{3}{8}\right], \\ \eta \cos ^{2}\left(\frac{4 \pi x}{3}\right), & x \in] \frac{3}{8}, \frac{3}{4}[ \\ \eta, & x \in\left[\frac{3}{4}, 1\right] .\end{cases}
$$

It is easy to see that

$$
\Phi(w)=\frac{32}{27} \pi^{4} \eta^{2}
$$

Therefore, since $\theta<\frac{8}{3 \sqrt{3}} \pi^{2} \eta$, one has $\Phi(w)>r$.

From assumption (A1) we obtain

$$
\Psi(w)=\int_{0}^{1} F(x, w(x)) d x+\frac{\mu}{\lambda} G(w(1)) \geq \int_{\frac{3}{4}}^{1} F(x, \eta) d x+\frac{\mu}{\lambda} G(\eta) .
$$

So, we have

$$
\frac{\Psi(w)}{\Phi(w)} \geq \frac{\int_{\frac{3}{4}}^{1} F(x, \eta) d x+\frac{\mu}{\lambda} G(\eta)}{\frac{32}{27} \pi^{4} \eta^{2}}
$$

Hence, if $G(\eta) \geq 0$, we find

$$
\frac{\Psi(w)}{\Phi(w)}>\frac{1}{\lambda}
$$


while if $G(\eta)<0$, the same relation holds since

$$
\mu G(\eta)>\frac{32}{27} \pi^{4} \eta^{2}-\lambda \int_{\frac{3}{4}}^{1} F(x, \eta) d x .
$$

Now, taking into account (3.4) and (3.6) results in

$$
\frac{\Psi(w)}{\Phi(w)}>\frac{1}{\lambda}>\frac{\sup _{\Phi(u) \leq r} \Psi(u)}{r},
$$

and condition $\left(a_{1}\right)$ of Lemma 2.1 is verified.

Now, in order to prove the coercivity of the functional $\Phi-\lambda \Psi$, first we assume that

$$
\limsup _{|\xi| \rightarrow+\infty} \frac{\sup _{x \in[0,1]} F(x, \xi)}{\xi^{2}}>0 .
$$

Therefore, fix

$$
\limsup _{|\xi| \rightarrow+\infty} \frac{\sup _{x \in[0,1]} F(x, \xi)}{\xi^{2}}<\varepsilon<\frac{F^{\theta}}{2 \theta^{2}}
$$

from (A3) there is a function $h_{\varepsilon} \in L^{1}([0,1])$ such that

$$
F(x, \xi) \leq \varepsilon \xi^{2}+h_{\varepsilon}(x)
$$

for each $x \in[0,1]$ and $\xi \in \mathbb{R}$. Taking (2.1) into account and since $\lambda<\frac{\theta^{2}}{2 F^{\theta}}$, it follows that

$$
\begin{aligned}
\lambda \int_{0}^{1} F(x, u(x)) d x & \leq \lambda\left(\varepsilon \int_{0}^{1}(u(x))^{2} d x+\int_{0}^{1} h_{\varepsilon}(x) d x\right) \\
& <\frac{\theta^{2}}{2 F^{\theta}}\left(\varepsilon \int_{0}^{1}(u(x))^{2} d x+\int_{0}^{1} h_{\varepsilon}(x) d x\right) \\
& \leq \frac{\theta^{2}}{2 F^{\theta}}\left(\varepsilon\|u\|^{2}+\left\|h_{\varepsilon}\right\|_{L^{1}([0,1])}\right)
\end{aligned}
$$

for each $u \in X$. Moreover, since $\mu<\bar{\delta}$, we obtain

$$
\limsup _{|\xi| \rightarrow+\infty} \frac{G(\xi)}{\xi^{2}}<\frac{1}{4 \mu}
$$

Thus, there is a positive constant $h_{\mu}$ such that

$$
G(\xi) \leq \frac{1}{4 \mu} \xi^{2}+h_{\mu}
$$

for each $\xi \in \mathbb{R}$. Thus, taking again (2.1) into account, it follows that

$$
\begin{aligned}
G(u(1)) & \leq \frac{1}{4 \mu}(u(1))^{2}+h_{\mu} \\
& \leq \frac{1}{4 \mu}\|u\|^{2}+h_{\mu}
\end{aligned}
$$


for each $u \in X$. Finally, putting together (3.7) and (3.8), we have

$$
\begin{aligned}
\Phi(u)-\lambda \Psi(u) & \geq \frac{1}{2}\|u\|^{2}-\frac{\theta^{2}}{2 F^{\theta}}\left(\varepsilon\|u\|^{2}+\left\|h_{\varepsilon}\right\|_{L^{1}([0,1])}\right)-\frac{1}{4}\|u\|^{2}-\mu h_{\mu} \\
& =\frac{1}{2}\left(\frac{1}{2}-\frac{\theta^{2}}{F^{\theta}} \varepsilon\right)\|u\|^{2}-\frac{\theta^{2}\left\|h_{\varepsilon}\right\|_{L^{1}([0,1])}}{2 F^{\theta}}-\mu h_{\mu} .
\end{aligned}
$$

On the other hand, if

$$
\limsup _{|\xi| \rightarrow+\infty} \frac{\sup _{x \in[0,1]} F(x, \xi)}{\xi^{2}} \leq 0,
$$

there exists a function $h_{\varepsilon} \in L^{1}([0,1])$ such that $F(x, \xi) \leq h_{\varepsilon}(x)$ for each $x \in[0,1]$ and $\xi \in \mathbb{R}$, and arguing as before we obtain

$$
\Phi(u)-\lambda \Psi(u) \geq \frac{1}{4}\|u\|^{2}-\frac{\theta^{2}\left\|h_{\varepsilon}\right\|_{L^{1}([0,1])}}{2 F^{\theta}}-\mu h_{\mu} .
$$

Both cases lead to the coercivity of $\Phi-\lambda \Psi$, and condition $\left(\mathrm{a}_{2}\right)$ of Lemma 2.1 is verified.

Since from (3.4) and (3.6)

$$
\lambda \in \Lambda \subseteq] \frac{\Phi(w)}{\Psi(w)}, \frac{r}{\sup _{\Phi(u) \leq r} \Psi(u)}[
$$

Lemma 2.1 ensures the existence of at least three critical points for the functional $\Phi-\lambda \Psi$, and the proof is complete.

Theorem 3.2 Let $\theta_{1}, \theta_{2}$, and $\eta$ be positive constants such that $\frac{3}{4} \sqrt{\frac{3}{2}} \frac{\theta_{1}}{\pi^{2}}<\eta<\frac{3}{8} \sqrt{\frac{3}{2}} \frac{\theta_{2}}{\pi^{2}}$ and $f:[0,1] \times \mathbb{R} \rightarrow \mathbb{R}$ be a mapping for which $f(x, t) \geq 0$ for every $(x, t) \in[0,1] \times\left[0, \theta_{2}\right]$. Assume that

(B1)

$$
\frac{\int_{0}^{1} F\left(x, \theta_{1}\right) d x}{\theta_{1}^{2}}<\frac{64}{81} \frac{\pi^{4} \int_{\frac{3}{4}}^{1} F(x, \eta) d x}{\eta^{2}}
$$

$$
\frac{\int_{0}^{1} F\left(x, \theta_{2}\right) d x}{\theta_{2}^{2}}<\frac{32}{81} \frac{\pi^{4} \int_{\frac{3}{4}}^{1} F(x, \eta) d x}{\eta^{2}} .
$$

Then, for each

$$
\left.\lambda \in \Lambda^{\prime}:=\right] \frac{32}{18} \frac{\pi^{4} \eta^{2}}{\int_{\frac{3}{4}}^{1} F(x, \eta) d x}, \frac{1}{2} \min \left\{\frac{\theta_{1}^{2}}{\int_{0}^{1} F\left(x, \theta_{1}\right) d x}, \frac{\theta_{2}^{2}}{2 \int_{0}^{1} F\left(x, \theta_{2}\right) d x}\right\}[
$$

and for every nonpositive continuous function $g: \mathbb{R} \rightarrow \mathbb{R}$, there exists $\delta^{*}>0$, where

$$
\delta^{*}:=\min \left\{\frac{\theta_{1}^{2}-2 \lambda \int_{0}^{1} F\left(x, \theta_{1}\right) d x}{2 G^{\theta_{1}}}, \frac{\theta_{2}^{2}-4 \lambda \int_{0}^{1} F\left(x, \theta_{2}\right) d x}{4 G^{\theta_{2}}}\right\},
$$


such that, for all $\mu \in\left[0, \delta^{*}\left[\right.\right.$, problem (1.1) admits at least three distinct weak solutions $u_{i}$ for $i \in\{1,2,3\}$ such that $0 \leq\left\|u_{i}\right\|_{\infty}<\theta_{2}$ for every $i \in\{1,2,3\}$.

Proof Without loss of generality, we can assume $f(x, t) \geq 0$ for every $(x, t) \in[0,1] \times \mathbb{R}$. Fix $\lambda, \mu$, and $g$ as in the conclusion and take $\Phi$ and $\Psi$ as in the proof of Theorem 3.1. Arguing as in the proof of Theorem 3.1, we observe that the regularity assumptions of Lemma 2.2 on $\Phi$ and $\Psi$ are satisfied. Then, our aim is to verify $\left(b_{1}\right)$ and $\left(b_{2}\right)$.

To this end, put $w$ as given in (3.5), and let $r_{1}=\frac{\theta_{1}^{2}}{2}$ and $r_{2}=\frac{\theta_{2}^{2}}{2}$. It is obvious that $2 r_{1}<$ $\Phi(w)<\frac{r_{2}}{2}$. It follows from $\mu<\delta^{*}$ and $G_{\eta}=0$ that

$$
\begin{aligned}
\frac{\sup _{\Phi(u)<r_{1}} \Psi(u)}{r_{1}} & =\frac{\sup _{\Phi(u)<r_{1}}\left(\int_{0}^{1} F(x, u(x)) d x+\frac{\mu}{\lambda} G(u(1))\right)}{r_{1}} \\
& \leq \frac{2 \int_{0}^{1} F\left(x, \theta_{1}\right) d x+\frac{2 \mu}{\lambda} G^{\theta_{1}}}{\theta_{1}^{2}} \\
& <\frac{1}{\lambda}<\frac{2}{3} \frac{\int_{\frac{3}{4}}^{1} F(x, \eta) d x+\frac{\mu}{\lambda} G_{\eta}}{\frac{32}{27} \pi^{4} \eta^{2}} \leq \frac{2}{3} \frac{\Psi(w)}{\Phi(w)} .
\end{aligned}
$$

Similarly,

$$
\begin{aligned}
\frac{2 \sup _{\Phi(u)<r_{2}} \Psi(u)}{r_{2}} & \leq \frac{4 \int_{0}^{1} F\left(x, \theta_{2}\right) d x}{\theta_{2}^{2}}+\frac{4 \mu}{\lambda} \frac{G^{\theta_{2}}}{\theta_{2}^{2}} \\
& <\frac{1}{\lambda}<\frac{2}{3} \frac{\int_{\frac{3}{4}}^{1} F(x, \eta) d x+\frac{\mu}{\lambda} G_{\eta}}{\frac{32}{27} \pi^{4} \eta^{2}} \leq \frac{2}{3} \frac{\Psi(w)}{\Phi(w)} .
\end{aligned}
$$

This implies that $\left(\mathrm{b}_{1}\right)$ and $\left(\mathrm{b}_{2}\right)$ of Lemma 2.2 are verified.

Finally, we verify that assumption $\left(\mathrm{b}_{3}\right)$ of Lemma 2.2 holds. Let $u_{1}$ and $u_{2}$ be two local minima for $\Phi-\lambda \Psi$. Then, $u_{1}$ and $u_{2}$ are critical points for $\Phi-\lambda \Psi$, and so they are weak solutions for problem (1.1). We claim that the weak solutions obtained are nonnegative. Indeed, if $u_{0}$ is a weak solution of problem (1.1), then one has

$$
\int_{0}^{1} u_{0}^{\prime \prime}(x) v^{\prime \prime}(x) d x=\lambda \int_{0}^{1} f\left(x, u_{0}(x)\right) v(x) d x-\mu g\left(u_{0}(1)\right) v(1)
$$

for all $v \in X$. Arguing by a contradiction, assume that the set $A:=\left\{x \in[0,1]: u_{0}(x)<0\right\}$ is nonempty and of positive measure. Put $v_{0}:=\min \left\{0, u_{0}\right\}$. Clearly, $v_{0} \in X$. So, taking into account that $u_{0}$ is a weak solution and by choosing $v=v_{0}$, from our sign assumptions on the data, one has

$$
\int_{A}\left(u_{0}^{\prime \prime}(x)\right)^{2} d x=\lambda \int_{A} f\left(x, u_{0}(x)\right) u_{0}(x) d x-\mu g\left(u_{0}(1)\right) u_{0}(1) \leq 0 .
$$

Hence, $u_{0} \equiv 0$ on $A$ which is absurd. Then we deduce $u_{1}(x) \geq 0$ and $u_{2}(x) \geq 0$ for each $x \in[0,1]$. Thus, it follows that $s u_{1}+(1-s) u_{2} \geq 0$ for all $s \in[0,1]$, and that

$$
\Psi\left(s u_{1}+(1-s) u_{2}\right)=\int_{0}^{1} F\left(x, s u_{1}(x)+(1-s) u_{2}(x)\right) d x+\frac{\mu}{\lambda} G\left(s u_{1}(1)+(1-s) u_{2}(1)\right) \geq 0 .
$$


So, also $\left(\mathrm{b}_{3}\right)$ holds. From Lemma 2.2, for every

$$
\lambda \in] \frac{3}{2} \frac{\Phi(w)}{\Psi(w)}, \min \left\{\frac{r_{1}}{\sup _{\Phi(u)<r_{1}} \Psi(u)}, \frac{\frac{r_{2}}{2}}{\sup _{\Phi(u)<r_{2}} \Psi(u)}\right\}[
$$

the functional $\Phi-\lambda \Psi$ has at least three distinct critical points which are the solutions of problem (1.1) and the conclusion is achieved.

\section{Conclusion}

By using as the main tools two critical point theorems presented recently in the works [3] and [6], we proved two multiplicity properties (Theorems 3.1 and 3.2) that guarantee the existence of an open interval $] \lambda^{\prime}, \lambda^{\prime \prime}[$ and $\tilde{\delta}>0$ such that, for each $\lambda \in] \lambda^{\prime}, \lambda^{\prime \prime}[$ and for each $\mu \in[0, \tilde{\delta}[$, a class of fourth-order boundary value problems depending on parameters $\lambda$ and $\mu$ (problem (1.1)) admits at least three distinct weak solutions.

\section{Acknowledgements}

The authors express their gratitude to the anonymous referees for useful comments and remarks.

\section{Funding}

Not applicable.

Availability of data and materials

Not applicable.

\section{Declarations}

Competing interests

The authors declare that they have no competing interests.

Authors' contributions

All authors read and approved the final manuscript.

\section{Publisher's Note}

Springer Nature remains neutral with regard to jurisdictional claims in published maps and institutional affiliations.

Received: 1 December 2020 Accepted: 13 October 2021 Published online: 26 October 2021

\section{References}

1. Baraket, S., Rădulescu, V.: Combined effects of concave-convex nonlinearities in a fourth-order problem with variable exponent. Adv. Nonlinear Stud. 16, 409-419 (2016)

2. Bonanno, G.: Relations between the mountain pass theorem and local minima. Adv. Nonlinear Anal. 1, 205-220 (2012)

3. Bonanno, G., Candito, P.: Non-differentiable functionals and applications to elliptic problems with discontinuous nonlinearities. J. Differ. Equ. 244, 3031-3059 (2008)

4. Bonanno, G., Chinnì, A., Tersian, S.: Existence results for a two point boundary value problem involving a fourth-order equation. Electron. J. Qual. Theory Differ. Equ. 2015, 33, 1-9 (2015)

5. Bonanno, G., Di Bella, B.: A boundary value problem for fourth-order elastic beam equations. J. Math. Anal. Appl. 343, 1166-1176 (2008)

6. Bonanno, G., Marano, S.A.: On the structure of the critical set of non-differentiable functions with a weak compactness condition. Appl. Anal. 89, 1-10 (2010)

7. Bonanno, G., Molica Bisci, G.: Infinitely many solutions for a boundary value problem with discontinuous nonlinearities. Bound. Value Probl. 2009, 670675, 1-20 (2009)

8. Cabada, A., lannizzotto, A.: A note on a question of Ricceri. Appl. Math. Lett. 25, 215-219 (2012)

9. Cabada, A., Tersian, S: Multiplicity of solutions of a two point boundary value problem for a fourth-order equation. Appl. Math. Comput. 219, 5261-5267 (2013)

10. Demarque, R., Miyagaki, O.H.: Multiplicity of solutions of a two point boundary value problem for a fourth-order equation. Adv. Nonlinear Anal. 4, 135-151 (2015)

11. Hadjian, A., Ramezani, M.: Existence of infinitely many solutions for fourth-order equations depending on two parameters. Electron. J. Differ. Equ. 2017, 117, 1-10 (2017)

12. Heidarkhani, S., Ferrara, M., Salari, A., Azimbagirad, M.: A variational approach to perturbed elastic beam problems with nonlinear boundary conditions. Math. Rep. 18, 573-589 (2016) 
13. Heidarkhani, S., Gharehgazlouei, F.: Existence results for a boundary value problem involving a fourth-order elastic beam equation. J. Nonlinear Funct. Anal. 2019, Article ID 28 (2019)

14. Kamenskii, M., Petrosyan, G., Wen, C.-F.: An existence result for a periodic boundary value problem of fractional semilinear differential equations in a Banach space. J. Nonlinear Var. Anal. 5, 155-177 (2021)

15. Kamenskii, M., Voskovskaya, N., Zvereva, M.: On periodic oscillations of some points of a string with a nonlinear boundary condition. Appl. Set-Valued Anal. Optim. 2, 35-48 (2020)

16. Li, F., Zhang, Q., Liang, Z.: Existence and multiplicity of solutions of a kind of fourth-order boundary value problem. Nonlinear Anal. 62, 803-816 (2005)

17. Ma, T.F.: Positive solutions for a beam equation on a nonlinear elastic foundation. Math. Comput. Model. 39 1195-1201 (2004)

18. Ricceri, B.: A further three critical points theorem. Nonlinear Anal. 71, 4151-4157 (2009)

19. Song, Y.: A nonlinear boundary value problem for fourth-order elastic beam equations. Bound. Value Probl. 2014, 191 1-11 (2014)

20. Tersian, S., Chaparova, J.: Periodic and homoclinic solutions of extended Fisher-Kolmogorov equations. J. Math. Anal. Appl. 260, 490-506 (2001)

21. Yang, L., Chen, H., Yang, X.: The multiplicity of solutions for fourth-order equations generated from a boundary condition. Appl. Math. Lett. 24, 1599-1603 (2011)

\section{Submit your manuscript to a SpringerOpen ${ }^{\circ}$} journal and benefit from:

- Convenient online submission

Rigorous peer review

Open access: articles freely available online

High visibility within the field

- Retaining the copyright to your article

Submit your next manuscript at $\boldsymbol{~ s p r i n g e r o p e n . c o m ~}$ 